\title{
LUQ view and the FAST exam: helpful or a hindrance in the adult trauma patient?
}

\author{
K O’Brien *, U Stolz, L Stolz, S Adhikari \\ From 9th WINFOCUS World Congress on Ultrasound in Emergency and Critical Care \\ Hong Kong. 6-9 November 2013
}

\section{Background}

A rapid, accurate FAST exam is essential to the adult trauma patient evaluation. Preliminary clinical observations suggest that adequate left upper quadrant (LUQ) views are difficult for novice sonographers to obtain and rarely contribute information that cannot be gleaned from other views.

\section{Objective}

We attempted to determine the proportion of positive FAST exams with adequate LUQ views, the proportion of these with novel information, and describe the most likely location of free fluid (FF) within the LUQ.

\section{Patients and methods}

This was a retrospective review of ultrasound images from a QA database (Qpath) of all positive patient care FAST exams from adult trauma patients at 2 urban, academic emergency departments over 2 years. Eligible studies were reviewed for location of intra-abdominal FF. FF seen in LUQ was further characterized as in splenorenal fossa, left paracolic gutter, and/or subdiaphragmatic/suprasplenic space. Reviewers then determined the frequency resident physicians obtained LUQ images deemed adequate for medical decision making. Data are reported as proportions with $95 \%$ confidence intervals (CIs, Jeffreys method).

\section{Results}

100 positive patient care FAST exams from adult trauma patients met eligibility criteria. While $32.0 \%$ (95\% CI: $23.5-41.6 \%)$ of positive FAST exams had FF in the LUQ, only $7.0 \%$ (95\% CI: 3.2-13.3\%) of patients with positive FAST exam had FF isolated to LUQ. Of all patients with FF in the LUQ $(n=32), 84 \%$ of patients
( $\mathrm{n}=27,95 \%$ CI: 69.1-93.8) had fluid in the paracolic gutter, with or without FF elsewhere in LUQ,.None (95\% CI: 0-7.5) had fluid seen only in splenorenal fossa, $3 \%(\mathrm{n}=1,95 \%$ CI: $0.3-13.7)$ had FF only above spleen. $51.0 \%$ of patients (95\% CI: $41.3-60.7 \%$ ) had LUQ views deemed inadequate for medical decision-making.

\section{Conclusion}

FF isolated to the LUQ occurs with a clinically relevant frequency in adult trauma patients. We propose physicians specifically obtain images of the left paracolic gutter/inferior splenic tip, as fluid in LUQ is most likely to accumulate here and is a relatively easy image to acquire for novice sonographers.

Published: 31 January 2014

\section{References}

1. Brown LD, Cai TT, DasGupta A: Interval estimation for a binomial proportion. Statistical Science 2001, 16:101-133.

2. Ma OJ, Mater JR, et al: Prospective analysis of a rapid trauma ultrasound examination performed by emergency physicians. J Trauma 1995, 38:879-885.

3. Laselle, et al: False-negative FAST examination: associations with injury characteristics and patient outcomes. Annals Emerg Medicine 2012, 60(3):326-34.e3.

4. Von Kuenssberg, et al: Sensitivity in detecting free intraperitoneal fluid with the pelvic views of the FAST exam. Am J Emerg Med 2003, 21(6):476-8.

5. Branney SW, Wolfe RE, Moore EE, et al: Quantitative sensitivity of ultrasound in detecting free intraperitoneal fluid. J Trauma 1995, 39:375-380.

doi:10.1186/2036-7902-6-S1-A3

Cite this article as: O'Brien et al:: LUQ view and the FAST exam: helpful or a hindrance in the adult trauma patient? Critical Ultrasound Journal 2014 6(Suppl 1):A3. 